\title{
Surgical Resection of Atypical Metastatic Melanoma to the Atriocaval Junction: A Case Report
}

\author{
Vitalis Osuji ${ }^{1}$, Maha Alkhuziem ${ }^{1}$, and Daniel Beckman ${ }^{1}$ \\ ${ }^{1}$ Indiana University School of Medicine
}

July 27, 2021

\begin{abstract}
Metastatic tumors to the heart include lymphomas, sarcoma, leukemia, and melanoma. Of these, metastatic melanoma is the most common, and are usually hematogenous, where metastatic lesions can be present in the left ventricle, right atrium, and/ or the right ventricle. We present an atypical metastatic melanoma pattern, where cardiac metastasis originated by direct extension from the adjacent gastric mesentery. We also present our trans-cardiac resection approach, which was the safest way to access the cardiac metastasis intra-operatively without disrupting the tumor.
\end{abstract}

Title: Surgical Resection of Atypical Metastatic Melanoma to the Atriocaval Junction: A Case Report.

Osuji, VC, Alkhuziem, MM, Beckman, DJ

Vitalis C. Osuji ${ }^{1}$ (Corresponding Author)

M.D, MPH Student, Class of 2022

Indiana University School of Medicine

340 West $10^{\text {th }}$ Street,

Fairbanks Hall, Suite 6200

Indianapolis, IN 46202-3082 USA

317-274-8157 | vosuji@iu.edu

Maha M. Alkhuziem, M.D . ${ }^{2}$

Cardiovascular Surgery Fellow,

Indiana University School of Medicine

340 West $10^{\text {th }}$ Street,

Fairbanks Hall, Suite 6200

Indianapolis, IN 46202-3082 USA

317-274-8157 | malkhuzi@iu.edu

Daniel J. Beckman, M.D. ${ }^{3}$

Professor of Clinical Surgery,

Indiana University School of Medicine 
340 West $10^{\text {th }}$ Street,

Fairbanks Hall, Suite 6200

Indianapolis, IN 46202-3082 USA

317-274-8157 | danbeckman@indy.rr.com

\begin{abstract}
:
Metastatic tumors to the heart include lymphomas, sarcoma, leukemia, and melanoma. Of these, metastatic melanoma is the most common, and are usually hematogenous, where metastatic lesions can be present in the left ventricle, right atrium, and/ or the right ventricle. We present an atypical metastatic melanoma pattern, where cardiac metastasis originated by direct extension from the adjacent gastric mesentery. We also present our trans-cardiac resection approach, which was the safest way to access the cardiac metastasis intra-operatively without disrupting the tumor.
\end{abstract}

\title{
Introduction :
}

Melanoma is a highly aggressive and unpredictable disease. It can metastasize to the heart as often as $64 \%$ in cases with advanced disease ${ }^{[\mathbf{1 - 2}]}$. Other sites of metastasis include the liver, bone, and brain ${ }^{[\mathbf{3}]}$. Once metastasis to other organs occur, malignant melanoma is defined as stage IV, with a poor prognosis. In addition to melanoma, other metastatic tumors to the heart include lymphoma, sarcoma, and leukemia ${ }^{[4]}$ - Cardiac metastasis from malignant melanoma occurs in extensive disease and is a late manifestation of disseminated disease. Some clinical presentations of metastatic melanoma to the heart include dysrhythmia, myocardial dysfunction, pericardial effusion, and heart failure. Long-term survival depends on various factors such as tumor stage, response to systemic treatments, surgical options, and pre-existing comorbidities. Therefore, early diagnosis of cardiac metastasis is desirable, as this could improve prognosis and could be a necessary step in averting morbidity and mortality from cardiac failure. Studies ${ }^{[5]}$ have found that the most common locations of metastatic melanoma to the heart include the left ventricle (41.9\%), right atrium $(35.5 \%)$, and right ventricle (19.4\%). In this report, we present a case of an atypical metastatic pattern of melanoma to the heart, as well as our trans-cardiac approach to surgical excision.

\section{Case Report :}

Our case is a 54-year-old Caucasian male with a history of melanoma, initially diagnosed in 2006. He was in a motor vehicle accident in 2015, which led to an evaluation with an abdominal computed tomographic (CT) scan that showed a liver mass. Upon further evaluation with fluorodeoxyglucose positron emission tomographic (PET) scan in January 2016, demonstrated abnormal uptake in the caudate lobe of the liver. After biopsy of two peritoneal lymph nodes and a diaphragmatic lesion which were positive for metastatic melanoma an extensive abdominal procedure was planned. The patient was evaluated by ophthalmology, and dermatology and no cutaneous or ocular source of primary melanoma was identified. He was started on immunotherapy. In 2017, he underwent surgery involving resection of the medial liver mass, with a left lobe hepatectomy, partial right hepatectomy, esophageal gastrectomy (Ivor Lewis Procedure), cholecystectomy, and retrohepatic Inferior Vena Cava (IVC) resection. Following the operation, he lost a significant amount of weight but was otherwise doing well.

In February 2021, he underwent a PET scan which showed a hypermetabolic lesion at the inferior cavoatrial junction, with no evidence of additional disease recurrence or progression. He underwent Endoscopic Ultrasound (EUS) and biopsy, which demonstrated malignant melanoma. He was referred to cardiothoracic surgery for possible resection. A transesophageal echocardiogram (TEE) indicated an ejection fraction (EF) of $66 \%$ and confirmed the intraatrial component of the tumor. His cardiac catherization results were pertinent for luminal irregularities of the distal left anterior descending, and 30\% stenosis of the mid left anterior descending. Cardiac Magnetic Resonance Imaging (MRI) showed a right atrial mass at the inferior cavoatrial junction with possible extra-cardiac extension. 
An operation was performed. The patient was placed under general anesthesia and placed on cardiopulmonary with a single atrial cannula and cooled to 20 degrees C. Following the removal of the atrial canula, an incision was made in the atrium along the sulcus terminalis just below the area of the sinus node down towards the IVC. After the atrium was opened, the point of adherence to the posterior atrial wall was identified and resected. The tumor extended by a pedicle into the mesentery of the gastric pull-up (figure 1 ). The tumor was completely resected, along with the adjacent heart and mesenteric tissues. The edges of the atrial tissues were cryoablated to eliminate any micro-invasion. The circulatory arrest time was less than 40 minutes. Pathologic examination revealed a $1.6 \times 1.5 \times 0.9 \mathrm{~cm}$ and $4.4 \times 1.8 \times 1.7 \mathrm{~cm}$ sized tumor, which was black in color (figure 2 ). A diagnosis of uveal type of melanoma was suspected.

The patient's postoperative course was uneventful. He was discharged from the hospital on the $5^{\text {th }}$ postoperative day and was referred to oncologic cardiology for continued immunotherapy. He would return to the clinic in two weeks for post-op follow-up in satisfactory condition.

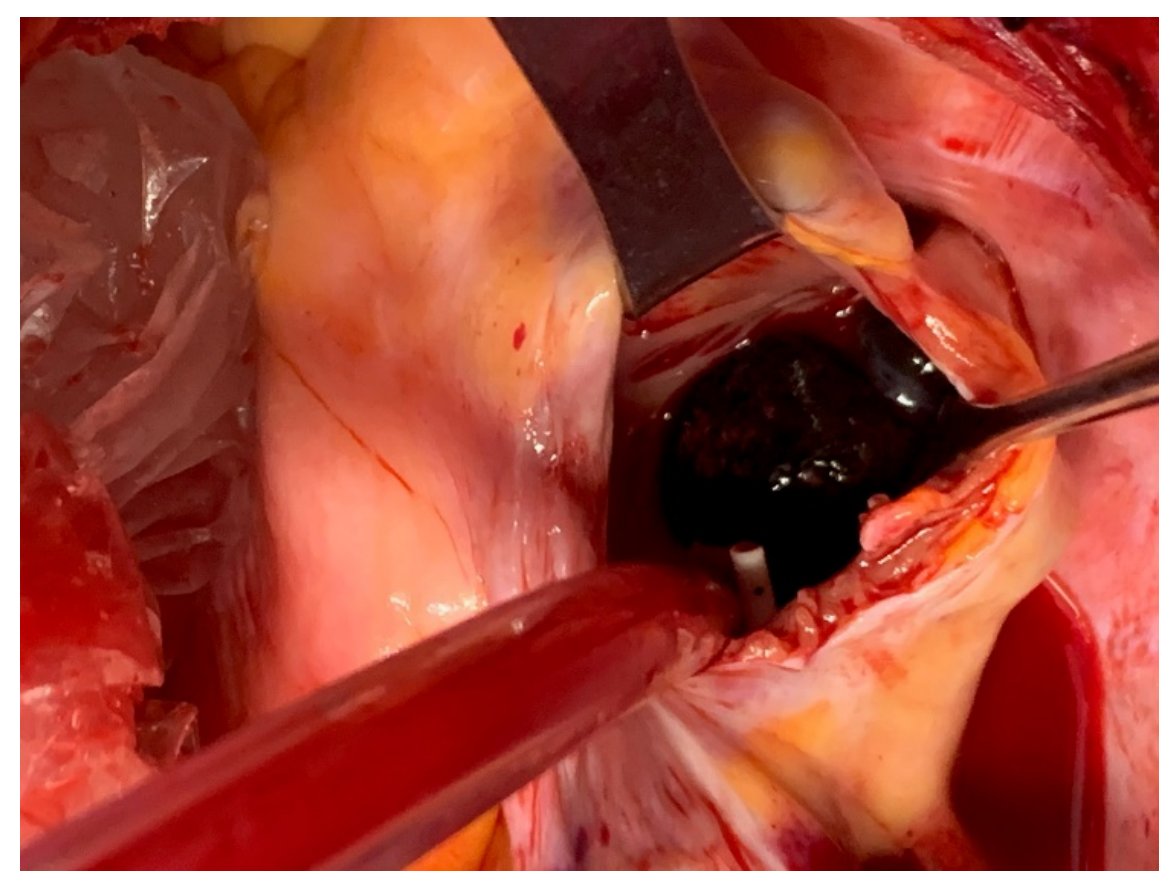

Figure 1 : Intraoperative photograph showing metastatic melanoma that adheres to the atriocaval junction of the heart. 


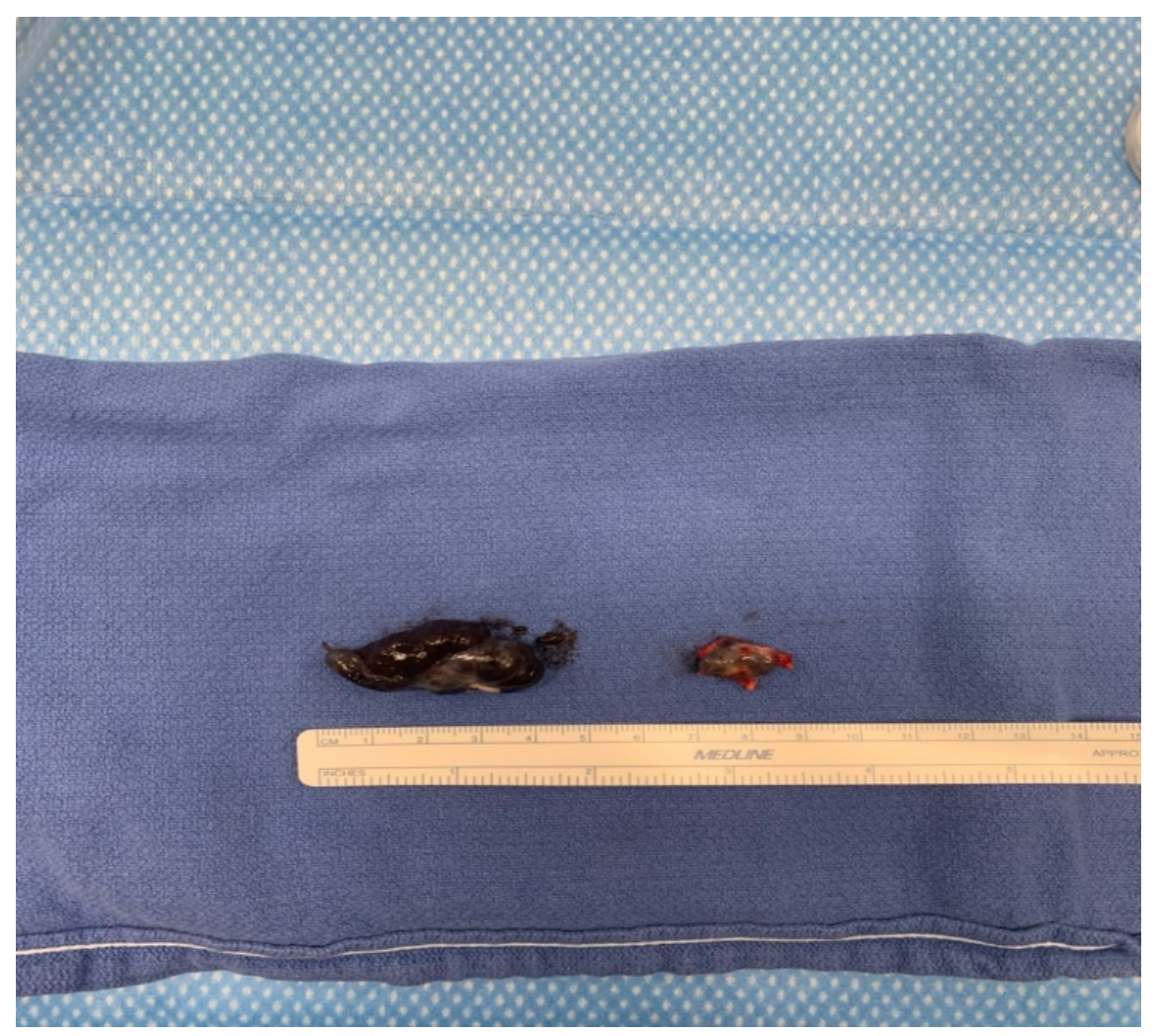

Figure 2 : Macroscopic view of metastatic melanoma.

\section{Discussion :}

This patient's past surgical history is pertinent for esophageal gastrectomy (Ivor Lewis technique) performed for resection of metastatic melanoma. Intra-operatively, the tumor extended by pedicle into the gastric pull up's mesentery implying that the cardiac metastasis most likely originated by direct extension from the adjacent gastric mesentery. The mesenteric portion of the resected specimen contained surgical staples, which further supports the possibility of direct extension into the cardiac structures. Cardiac metastases of melanoma to the heart are usually hematogenous ${ }^{[6]}$. They commonly present as multifocal lesions in the left ventricle, right atrium, and/ or the right ventricle. However, our patient's tumor presented as a solitary metastasis to the right atrial caval junction. Trans-cardiac resection of the tumor was affected with deep hypothermic circulatory arrest without complications. We felt this was the safest way to access the metastasis at the atrial caval junction without disrupting the tumor.

IRB Approval and Consent : N/A

Prior to journal submission, we received confirmation from the Indiana University IRB and compliance officer that IRB submission and approval is not required for this case study. However, verbal consent was granted by patient prior to hospital discharge.

Funding : N/A

None - not applicable

Conflict of Interest : N/A

None - not applicable

\section{References:}


[1] Allen, BC, et al. Metastatic Melanoma to the Heart . Current Problems in Diagnostic Radiology. 2012: 41(5); $159-164$.

[2] Zitzelsberger, T. et al. Imaging Characteristics of Cardiac Metastases in patients with Malignant Melanoma . Cancer Imaging. 2017: 19 (17)

[3] Damsky, WE, et al. Decoding Melanoma Metastasis . Cancers. 2011: 3(1); 126 - 163

[4] Goldberg, AD, et al. Tumors Metastatic to the Heart. Circulation. 2013; 128: 1790 - 1794

[5] Balinski, AM, et al. Metastatic Melanoma of the Heart: A Systematic Review. 2020: 38(15)

[6] Shapiro, LM, et al. Cardiac Diagnosis and Management. Heart. 2001; 85: $218-222$ 